\title{
FUNCTIONS IN THE FRESNEL CLASS
}

\author{
K. S. CHANG, G. W. JOHNSON AND D. L. SKOUG
}

\begin{abstract}
Let $H$ be a separable infinite-dimensional Hilbert space over $\mathbf{R}$. The Fresnel class $\mathcal{F}(H)$ of $H$ consists of all Fourier-Stieltjes transforms of bounded Borel measures on $H$. There are several results insuring that various functions of interest in connection with the Feynman integral and quantum mechanics are in $\mathcal{F}(H)$. We give a theorem which has most of these results as corollaries as well as many further corollaries involving the reproducing kernel Hilbert spaces of Gaussian stochastic processes.
\end{abstract}

1. Introduction. Let $M(H)$ denote the space of all complex-valued (or $\mathbf{C}$ valued) countably additive measures defined on $B(H)$, the Borel class of $H$. A C-valued countably additive measure $\mu$ necessarily has finite total variation $\|\mu\|$ $[30$, p. 119]. Under the norm $\|\cdot\|$ and with convolution as multiplication, $M(H)$ is a commutative Banach algebra with identity $[3, \mathrm{p} .17]$ which we will refer to as the measure algebra of $H$. The space $\mathcal{F}(H)$ of all Fresnel integrable functions (or the Fresnel class of $H$ ) is defined [3, p. 17] as the space of all Fourier transforms of elements of $M(H)$; that is, $f: H \rightarrow \mathbf{C}$ is in $\mathcal{F}(H)$ if and only if there is a measure $\mu$ in $M(H)$ such that, for all $h$ in $H$,

$$
f(h)=\int_{H} \exp \left\{i\left(h, h_{1}\right)\right\} d \mu\left(h_{1}\right),
$$

or, briefly, $f=\hat{\mu}$. The correspondence $\mu \rightarrow f=f_{\mu}$ is injective and preserves the algebraic operations $[3$, pp. 17, 18] converting convolution into pointwise multiplication as one expects of a transform. Thus if we define $\left\|f_{\mu}\right\|:=\|\mu\|, \mathcal{F}(H)$ becomes a Banach algebra isomorphic to $M(H)$. Although it will not be the direct concern of this paper, we note that the Fresnel integral of $f_{\mu}$ is defined $[3$, p. 18] by

$$
\mathcal{F}\left(f_{\mu}\right)=\int_{H} \exp \left\{\frac{-i}{2}\|h\|^{2}\right\} d \mu(h) .
$$

When appropriate choices are made for the Hilbert space $H$ and the function $f_{\mu}$, the Fresnel integral provides one means of giving a rigorous definition of Feynman's path integral from quantum theory $[3,13]$. In fact, since the influential monograph of Albeverio and Høegh-Krohn [3] in 1976, most of the mathematically rigorous work on the Feynman integral has involved, in one way or another, the assumption that functions under consideration are Fourier transforms of bounded

Received by the editors March 24, 1986.

1980 Mathematics Subject Classification (1985 Revision). Primary 81C30, 60G15; Secondary 42B10, 28C20, 81 C35.

Research of the first author was partially supported by the Korean Science and Engineering Foundation. The research of the second and third authors was partially supported by NSF grant DMS 8403197. 
Borel measures. The following papers and references contained therein provide a sample of this work: $[1,2,4-12,15,18-20,23,24,31]$. Transform approaches to the Feynman integral have their limitations as pointed out in [21] but also many notable advantages. In most (not all) respects the Feynman integral is a simpler, more fully developed, and better unified subject under transform assumptions $[\mathbf{1 2}$, 24].

In order to see that the Fresnel integral is relevant to the Feynman integral and quantum theory, it is essential to show that various functions of interest in this context belong to $\mathcal{F}(H)$. Several such results are known. The purpose of this paper is to prove a theorem which has most of these results as corollaries as well as many further corollaries involving various Gaussian stochastic processes.

2. Functions in $\mathcal{F}(H)$ : A general theorem. After the statement of the theorem, in order to better understand the motivation, some readers may wish to go directly to the corollaries in the following section. $\mathbf{R}$.

THEOREM 1. (1) Let $H$ be a separable infinite-dimensional Hilbert space over

(2) Let $(Y, Y, \eta)$ be a measure space, where $\eta$ is either a nonnegative, $\sigma$-finite measure or a $\mathbf{C}$-valued measure.

(3) Let $\phi_{i}: Y \rightarrow H$ be $Y-B(H)$-measurable for $i=1, \ldots, d$.

(4) Let $\theta: Y \times \mathbf{R}^{d} \rightarrow \mathbf{C}$ be given by $\theta(y ; \cdot)=\hat{\nu}_{y}(\cdot)$, where $\nu_{y}$ is in $M\left(\mathbf{R}^{d}\right)$, the measure algebra of $\mathbf{R}^{d}$, for every $y$ in $Y$ and where the family $\left\{\nu_{y}: y\right.$ in $\left.Y\right\}$ satisfies: (i) $\nu_{y}(B)$ is a $\mathcal{Y}$-measurable function of $y$ for every $B$ in $B\left(\mathbf{R}^{d}\right)$, and (ii) $\left\|\nu_{y}\right\|$ is in $L^{1}(Y, Y,|\eta|)$.

Under these hypotheses, $f: H \rightarrow \mathbf{C}$ given by

$$
f(h):=\int_{Y} \theta\left(y ;\left\langle\left(h, \phi_{1}(y)\right), \ldots,\left(h, \phi_{d}(y)\right)\right\rangle\right) d \eta(y)
$$

belongs to $\mathcal{F}(H)$ and satisfies the inequality

$$
\|f\| \leq \int_{Y}\left\|\nu_{y}\right\| d|\eta|(y) .
$$

Further, since $\mathcal{F}(H)$ is a Banach algebra, $g$ is in $\mathcal{F}(H)$ where

$$
g(h):=\exp (f(h)) \text {. }
$$

REMARKS. 1. It suffices to assume in (4) that $\theta(y ; \cdot)=\hat{\nu}_{y}(\cdot)$ for $\eta$-a.e. $y$ in $Y$. In some cases this hypothesis seems more natural. However, under this alternative, one must add the assumption that $\theta$ is measurable with respect to the product $\sigma$-algebra $y \times B\left(\mathbf{R}^{d}\right)$ whereas this can be proved under the present assumptions.

2. Since $\mathcal{F}(H)$ is a Banach algebra, many analytic functions of $f$ can be formed. We explicitly mention the exponential function in (2.3) because it plays a central role in the quantum theory.

In the proof of our theorem we will use the simple Unsymmetric Fubini Theorem discussed by Johnson in [16]:

THEOREM $2[\mathbf{1 6}]$. Let $(Y, Y, \eta)$ be a measure space, where $\eta$ is either a nonnegative, $\sigma$-finite measure or a $\mathbf{C}$-valued measure, and let $(Z, Z)$ be a measurable 
space. For $|\eta|$-a.e. $y$, let $\nu_{y}$ be a $\mathbf{C}$-valued measure on $(Z, Z)$. Suppose that for any $B$ in $Z, \nu_{y}(B)$ is a $Y$-measurable function of $y$. Then

A. [16, Proposition 1] for any $E$ in $\mathcal{Y} \times Z, \nu_{y}\left(E^{(y)}\right)$ is $\mathcal{Y}$-measurable where $E^{(y)}$ denotes the section of $E$ by $y$, and

B. [16, Proposition 2] for any bounded, $\mathbf{C}$-valued, $\mathcal{Y} \times Z$-measurable function $\theta$ on $Y \times Z, \int_{Z} \theta(y, z) d \nu_{y}(z)$ is a $y$-measurable function of $y$.

If we add the assumption that $\left\|\nu_{y}\right\| \leq h(y)$, where $h$ is in $L_{1}(Y, Y,|\eta|)$, and define $\rho$ on $\mathcal{Y} \times Z$ by

$$
\rho(E):=\int_{Y} \nu_{y}\left(E^{(y)}\right) d \eta(y)
$$

then [16, Theorem]

C. $\rho$ is a C-valued, countably additive measure on $\mathcal{Y} \times Z$ with $\|\rho\| \leq\|h\|_{1}$, and

D. If $\theta(y, z)$ is bounded and $\mathcal{Y} \times Z$-measurable, then $\int_{Z} \theta(y, z) d \nu_{y}(z)$ is in $L_{1}(Y, \mathcal{Y},|\eta|)$ and we have

$$
\int_{Y}\left[\int_{Z} \theta(y, z) d \nu_{y}(z)\right] d \eta(y)=\int_{Y \times Z} \theta(y, z) d \rho(y, z) .
$$

REMARKS. 1. In [16], $\eta$ was required to be a nonnegative, $\sigma$-finite measure. However, a careful check of the proof shows that a $\mathbf{C}$-valued measure $\eta$ can also be allowed.

2. If $\nu_{y}=\nu$ is independent of $y$, then $\rho$ is just $\eta \times \nu$ and Theorem 2 is a part of the ordinary Fubini Theorem.

PROOF OF THEOREM 1. We begin by considering questions of measurability. First we clarify assumption (4)(ii) by showing that $\left\|\nu_{y}\right\|$ is measurable as a function of $y$. Let $C_{0}\left(\mathbf{R}^{d}\right)$ denote the space of continuous functions on $\mathbf{R}^{d}$ which vanish at $\infty$. It is well known that $M\left(\mathbf{R}^{d}\right)$ can be identified with the dual of the Banach space $\left(C_{0}\left(\mathbf{R}^{d}\right),\|\cdot\|_{\infty}\right)\left[30\right.$, p. 131]. Given $\alpha$ in $C_{0}\left(\mathbf{R}^{d}\right)$, let $\beta(y)=\beta_{\alpha}(y):=$ $\int_{\mathbf{R}^{d}} \alpha(v) d \nu_{y}(v)$. B of Theorem 2 implies that $\beta_{\alpha}(y)$ is measurable as a function of $y$. Now take $D$ to be a countable dense subset of the unit ball of $C_{0}\left(\mathbf{R}^{d}\right)$. Then $\left\|\nu_{y}\right\|=\sup \left\{\left|\beta_{\alpha}(y)\right|: \alpha \in D\right\}$, and so $\left\|\nu_{y}\right\|$ is the supremum of a countable collection of measurable functions of $y$ and so is itself measurable.

Next we claim that $\theta$ is $\mathcal{Y} \times B\left(\mathbf{R}^{d}\right)$-measurable. By assumption (4),

$$
\theta(y ; u)=\int_{\mathbf{R}^{d}} \exp (i u v) d \nu_{y}(v) .
$$

The measurability of $\theta$ follows from another application of $\mathrm{B}$ of Theorem 2 where the roles of $Y, \mathcal{Y}, \eta, Z$, and $Z$ from Theorem 2 are played by $Y \times \mathbf{R}^{d}, \mathcal{Y} \times B\left(\mathbf{R}^{d}\right)$, $|\eta| \times l$ (where $l$ denotes Lebesgue measure on $\mathbf{R}^{d}$ ), $\mathbf{R}^{d}$, and $B\left(\mathbf{R}^{d}\right)$, respectively. The function mapping $(y, u, v)$ to $\exp (i u v)$ serves as the $\theta$ from Theorem 2 and $\nu_{y}$ serves as $\nu_{y, u}$.

The fact that the integrand in (2.1) is measurable as a function of $y$ for every $h$ in $H$ now follows quite readily from assumption (3) and the measurability of $\theta$.

Our next step is to define the measure $\mu$ in $M(H)$ which we will eventually show satisfies $\hat{\mu}(h)=f(h)$. First we use Theorem 2, the family of measures $\left\{\nu_{y}: y\right.$ in $\left.Y\right\}$, and the measure $\eta$ to produce a measure $\rho$ on $\mathcal{Y} \times B\left(\mathbf{R}^{d}\right)$. Given $E$ in $\mathcal{Y} \times B\left(\mathbf{R}^{d}\right)$, 
let $\rho(E):=\int_{Y} \nu_{y}\left(E^{(y)}\right) d \eta(y)$ as in (2.4). Note that, by $\mathrm{C}$ of Theorem 2, $\rho$ satisfies

$$
\|\rho\| \leq \int_{Y}\left\|\nu_{y}\right\| d|\eta|(y) .
$$

Now define $\Phi: Y \times \mathbf{R}^{d} \rightarrow H$ by $\Phi\left(y,\left\langle v_{1}, \ldots, v_{d}\right\rangle\right):=v_{1} \phi_{1}(y)+\cdots+v_{d} \phi_{d}(y) . \Phi$ is easily seen to be $\mathcal{Y} \times B\left(\mathbf{R}^{d}\right)-B(H)$-measurable using hypothesis (3). Define $\mu$ as the image measure $\mu:=\rho \circ \Phi^{-1}$. Then $\mu$ is in $M(h)$ and $\|\mu\| \leq\|\rho\|$. Once we have shown that $f=\hat{\mu}$, this last inequality and (2.7) immediately imply (2.2).

It remains only to show that $\hat{\mu}(h)=f(h)$ for all $h$ in $H$. The third equality below follows from the Change of Variables Theorem; the sixth comes from formula (2.5) of the Unsymmetric Fubini Theorem.

$$
\begin{aligned}
\hat{\mu}(h) & =\int_{H} \exp \left\{i\left(h, h_{1}\right)\right\} d \mu\left(h_{1}\right)=\int_{H} \exp \left\{i\left(h, h_{1}\right)\right\} d\left(\rho \circ \Phi^{-1}\right)\left(h_{1}\right) \\
& =\int_{Y \times \mathbf{R}^{d}} \exp \left\{i\left(h, \Phi\left(y,\left\langle v_{1}, \ldots, v_{d}\right\rangle\right)\right)\right\} d \rho\left(y,\left\langle v_{1}, \ldots, v_{d}\right\rangle\right) \\
& =\int_{Y \times \mathbf{R}^{d}} \exp \left\{i\left(h, v_{1} \phi_{1}(y)+\cdots+v_{d} \phi_{d}(y)\right)\right\} d \rho\left(y,\left\langle v_{1}, \ldots, v_{d}\right\rangle\right) \\
& =\int_{Y \times \mathbf{R}^{d}} \exp \left\{i \left[\left\langle v_{1}, \ldots, v_{d}\right\rangle,\right.\right. \\
& \left.=\int_{Y}\left\{\int_{\mathbf{R}^{d}} \exp \left\{i\left[\left\langle v_{1}, \ldots, v_{d}\right\rangle,(y)\right), \ldots,\left(h, \phi_{d}(y)\right)\right\rangle\right]\right\} d \rho\left(y,\left\langle v_{1}, \ldots, v_{d}\right\rangle\right) \\
& =\int_{Y} \hat{\nu}_{y}\left(\left\langle\left(h, \phi_{1}(y)\right), \ldots,\left(h, \phi_{d}(y)\right)\right)\right\rangle d \eta(y) \\
& \left.\left.\left.\left.\left.=\int_{Y} \theta\left(y ;\left\langle\left(h, \phi_{1}(y)\right), \ldots,\left(h, \phi_{d}(y)\right)\right\rangle\right) d \eta(y)\right), \ldots,\left(h, \phi_{d}(y)\right)\right\rangle\right]\right\} d \nu_{y}\left(v_{1}, \ldots, v_{d}\right)\right\} d \eta(y) \\
& =f(h) .
\end{aligned}
$$

3. Corollaries. One particular Hilbert space and variations of it have been used in the applications of the Fresnel integral to nonrelativistic quantum mechanics. Suppose that $t>0$ and let $H_{1}=H_{1}[0, t]$ be the space of $\mathbf{R}$-valued functions $\gamma$ on $[0, t]$ which are absolutely continuous, which vanish at $t$, and whose derivative $D \gamma$ is in $L^{2}[0, t]$. The inner product on $H_{1}$ is given by

$$
(\gamma, \beta)_{H_{1}}:=\int_{0}^{t}(D \gamma)(s)(D \beta)(s) d s .
$$

For some problems, it is natural to consider an infinite interval such as $[0,+\infty)$. In this case, we would take $H_{1}[0,+\infty)$ to be the absolutely continuous functions $\gamma$ which vanish at 0 and such that $D \gamma$ is in $L^{2}[0,+\infty)$. Most of the corollaries which we will formally state will be in the setting of the Hilbert space $H_{1}=H_{1}[0, t]$. For simplicity, we will restrict our attention to one space dimension except for Corollary 3 where we will illustrate the handling of additional space dimensions. In 
spite of the emphasis on $H_{1}$, it will be clear from our last corollary and a variety of remarks that there are many further corollaries.

It will be helpful to introduce the family of functions $\left\{\gamma_{\tau}: 0 \leq \tau \leq t\right\}$ from $H_{1}$ :

$$
\gamma_{\tau}(s):= \begin{cases}t-\tau, & 0 \leq s \leq \tau, \\ t-s, & \tau \leq s \leq t .\end{cases}
$$

These functions have the reproducing property,

$$
\left(\gamma, \gamma_{\tau}\right)_{H_{1}}=\gamma(\tau) \quad \text { for all } \gamma \text { in } H_{1} \text {. }
$$

Let $\psi: \mathbf{R} \rightarrow \mathbf{C}$. Define $f_{1}: H_{1} \rightarrow \mathbf{C}$ by the formula

$$
f_{1}(\gamma):=\psi(\gamma(0))
$$

Functions of the form (3.4) are of interest in quantum mechanics where $\psi$ is the initial state of the quantum system. Our first corollary is due to Albeverio and Høegh-Krohn [3, p. 29]. It is a simple result, but it is interesting to see how it fits into the framework of Theorem 1.

COROLlaRY 1. If $\psi=\hat{\nu}$ where $\nu$ is in $M(\mathbf{R})$, then $f_{1}$ belongs to $\mathcal{F}\left(H_{1}\right)$.

ProOF. Apply Theorem 1 after making the following choices: $H=H_{1} ;(Y, Y, \eta)$ $=([0, t], B([0, t]), \eta)$ where $\eta$ is any probability measure; $d=1$ and $\phi_{1}(s) \equiv \gamma_{0}$ (see $(3.2)) ; \theta(s ; \cdot)=\hat{\nu}(\cdot)$. With these choices, the right-hand side of (2.1) becomes $\int_{0}^{t} \hat{\nu}\left(\left(\gamma, \gamma_{0}\right)_{H_{1}}\right) d \eta(s)=\psi(\gamma(0))$, and the result follows.

REMARK. It is natural to ask if conditions insuring that a function is in $\mathcal{F}(H)$ are necessary as well as sufficient. For a certain class of functions including $f_{1}$, the authors have shown earlier [7] that the answer is 'yes.' Specifically, if $f_{1}$ is given by (3.4), then $f_{1}$ is in $\mathcal{F}\left(H_{1}\right)$ if and only if $\psi=\hat{\nu}$ where $\nu$ is in $M(\mathbf{R})$.

Exponentials of functions of the type treated in our next corollary arise naturally in quantum mechanics where $\theta$ is the potential associated with the problem (see (2.3) in the statement of Theorem 1). This result is also due to Albeverio and Høegh-Krohn [3, p. 29].

Corollary 2. Let $\theta=\hat{\nu}$ where $\nu$ is in $M(\mathbf{R})$. Define $f_{2}: H_{1} \rightarrow \mathbf{C}$ by

$$
f_{2}(\gamma):=\int_{0}^{t} \theta(\gamma(\tau)) d \tau
$$

Then $f_{2}$ belongs to $\mathcal{F}\left(H_{1}\right)$.

Proof. Take $H, Y, y, d$, and $\theta$ as in the proof of Corollary 1 . Let $\eta$ be Lebesgue measure on $[0, t]$ and take $\phi_{1}(\tau)=\gamma_{\tau}$ as in (3.2). Then the right-hand side of $(2.1)$ is just

$$
\int_{0}^{t} \theta\left(\left(\gamma, \gamma_{\tau}\right)_{H_{1}}\right) d \tau=\int_{0}^{t} \theta(\gamma(\tau)) d \tau
$$

and the result follows.

Let $H_{1}^{n}=H_{1}^{n}[0, t]$ consist of functions $\gamma:[0, t] \rightarrow \mathbf{R}^{n}$ such that each component $\gamma_{j}$ is in $H_{1}$. Define the inner product of $\gamma$ and $\beta$ in $H_{1}^{n}$ as the sum of the $H_{1}$ inner products of the components. 
COROllary 3. Let $\theta=\hat{\nu}$ where $\nu$ is in $M\left(\mathbf{R}^{n}\right)$. Define $f_{3}: H_{1}^{n} \rightarrow \mathbf{C}$ by

$$
f_{3}(\gamma):=\int_{0}^{t} \theta\left(\gamma_{1}(\tau), \ldots, \gamma_{n}(\tau)\right) d \tau
$$

Then $f_{3}$ belongs to $\mathcal{F}\left(H_{1}^{n}\right)$.

ProOF. Apply Theorem 1 with $H=H_{1}^{n} ; Y, \mathcal{Y}$, and $\eta$ as in Corollary $2 ; \theta(\tau ; \cdot)=$ $\hat{\nu}(\cdot) ; d=n$, and $\phi_{j}(\tau)(j=1, \ldots, n)$ the function in $H_{1}^{n}$ which is 0 except in the $j$ th component where it is $\gamma_{\tau}$.

It is of interest to have a result similar to Corollaries 2 and 3 but for timedependent potentials. the next corollary is due to the second author [15, Corollary 1], but the analogous result in a Wiener space setting was done earlier by Cameron and Storvick [5] and given in somewhat improved form with a simpler proof by Johnson and Skoug [18, Theorem 1 and 20, Corollary 3.5].

CoRollary 4. Let $\theta:[0, t] \times \mathbf{R} \rightarrow \mathbf{C}$ be given by $\theta(\tau ; \cdot)=\hat{\nu}_{\tau}(\cdot)$ where $\nu_{\tau}$ is in $M(\mathbf{R})$ for every $\tau$ in $[0, t]$ and where the family $\left\{\nu_{\tau}: 0 \leq \tau \leq t\right\}$ satisfies: (i) $\nu_{\tau}(B)$ is a Borel measurable function of $\tau$ for every $B$ in $B(\mathbf{R})$, and (ii) $\left\|\nu_{\tau}\right\|$ is integrable over $[0, t]$ with respect to Lebesgue measure l. Define $f_{4}: H_{1} \rightarrow \mathbf{C}$ by

$$
f_{4}(\gamma):=\int_{0}^{t} \theta(\tau ; \gamma(\tau)) d \tau
$$

Then $f_{4}$ belongs to $\mathcal{F}\left(H_{1}\right)$.

ProOF. Make the choice of $H, Y$, etc. from Theorem 1 as in Corollary 2 except take $\theta(\tau ; \cdot)=\hat{\nu}_{\tau}(\cdot)$.

The results given in the next two corollaries were proved by the authors in 1983 but were not written up for publication then as we had begun to suspect that a more general theorem was possible. In the first of these corollaries all the choices are as in Corollary 4 except that Lebesgue measure is replaced by a general Borel measure $\eta$ on $[0, t]$. Recent work of Johnson and Lapidus [17] and Lapidus [27] shows that there are many reasons to consider such measures $\eta$.

COROLlaRY 5. Let the hypotheses of Corollary 4 be satisfied and let $\eta$ be a Borel measure on $[0, t]$. Define $f_{5}: H_{1} \rightarrow \mathbf{C}$ by

$$
f_{5}(\gamma):=\int_{0}^{t} \theta(\tau ; \gamma(\tau)) d \eta(\tau)
$$

Then $f_{5}$ belongs to $\mathcal{F}\left(H_{1}\right)$.

'Potentials' involving a double (or multiple) dependence on time are of interest. Such potentials arise in interacting systems when the coordinates associated with one particle are integrated out. This possibility was discussed in Feynman's original paper $[13, \S 13]$. There he formally integrated out the oscillator coordinates in a system involving a harmonic oscillator interacting with a particle moving in a potential.

CoROllaRY 6. Let $\theta:[0, t]^{2} \times \mathbf{R}^{2} \rightarrow \mathbf{C}$ be given by $\theta\left(\tau_{1}, \tau_{2} ; \cdot, \cdot\right)=\hat{\nu}_{\tau_{1}, \tau_{2}}(\cdot, \cdot)$ where $\nu_{\tau_{1}, \tau_{2}}$ is in $M\left(\mathbf{R}^{2}\right)$ for every $\left(\tau_{1}, \tau_{2}\right)$ in $[0, t]^{2}$ and where the family $\left\{\nu_{\tau_{1}, \tau_{2}}\right.$ : $\left(\tau_{1}, \tau_{2}\right)$ in $\left.[0, t]^{2}\right\}$ satisfies: (i) $\nu_{\tau_{1}, \tau_{2}}(B)$ is a Borel measurable function of $\left(\tau_{1}, \tau_{2}\right)$ 
for every $B$ in $B\left(\mathbf{R}^{2}\right)$, and (ii) $\left\|\nu_{\tau_{1}, \tau_{2}}\right\|$ is integrable over $[0, t]^{2}$ with respect to $|\eta|$ where $\eta$ is a Borel measure on $[0, t]^{2}$. Define $f_{6}: H_{1} \rightarrow \mathbf{C}$ by

$$
f_{6}(\gamma):=\int_{0}^{t} \int_{0}^{t} \theta\left(\tau_{1}, \tau_{2} ; \gamma\left(\tau_{1}\right), \gamma\left(\tau_{2}\right)\right) d \eta\left(\tau_{1}, \tau_{2}\right)
$$

Then $f_{6}$ belongs to $\mathcal{F}\left(H_{1}\right)$.

Proof. Apply Theorem 1 with $Y=[0, t]^{2}, y=B\left([0, t]^{2}\right), d=2, \phi_{1}\left(\tau_{1}, \tau_{2}\right)=$ $\gamma_{\tau_{1}}$, and $\phi_{2}\left(\tau_{1}, \tau_{2}\right)=\gamma_{\tau_{2}}$. The other choices are clear.

It follows as usual from Corollary 6 that $\exp \left(f_{6}\right)$ is in $\mathcal{f}\left(H_{1}\right)$. When this is combined with a recent result of the authors [8] on quadratic potentials, one sees that a rather large class of potentials involving two time parameters can be dealt with in this theory. This was already noted in [8, Corollary 4.7]. We remark that there is an analog of Corollary 6 for $n$ time parameters, and also that in a paper which has been submitted for publication [29], Park and Skoug have a result on quadratic potentials with $n$ time parameters.

In the next corollary, $H$ will be a general separable infinite-dimensional Hilbert space over $\mathbf{R}$ as in Theorem 1 . The result to follow was given by the authors in [7]. The main point of [7] was actually a converse result which will be remarked on following the proof of the corollary.

COROLlaRY 7. Let $\left\{h_{1}, \ldots, h_{d}\right\}$ be a finite subset of $H$ and let $\psi=\hat{\nu}$ where $\nu$ is in $M\left(\mathbf{R}^{d}\right)$. Define $f_{7}: H \rightarrow \mathbf{C}$ by

$$
f_{7}(h):=\psi\left(\left\langle\left(h, h_{1}\right), \ldots,\left(h, h_{d}\right)\right\rangle\right) .
$$

Then $f_{7}$ belongs to $\mathcal{F}(H)$.

ProOF. Let $(Y, y, \eta)$ be a probability space and, for $i=1, \ldots, d$, let $\phi_{i} \equiv h_{i}$. Take $\theta(y ; \cdot)=\psi(\cdot)=\hat{\nu}(\cdot)$. Then the right-hand side of $(2.1)$ equals the right-hand side of (3.10) and the result follows.

REMARK. If $\left\{h_{1}, \ldots, h_{d}\right\}$ is a linearly independent set, the authors have shown in [7] that $f_{7}$ given by $(3.10)$ is in $\mathcal{F}(H)$ if and only if $\psi=\hat{\nu}$ where $\nu$ is in $M\left(\mathbf{R}^{d}\right)$. In fact, they have a related result $[\mathbf{7}$, Corollary 12, p. 26$]$ where linear independence is not assumed.

We have concentrated our corollaries on the Hilbert space $H_{1}$. Now we make remarks indicating how further corollaries may be obtained and state one final corollary as an explicit example.

Hilbert spaces other than $H_{1}$ appeared in the monograph [3] in connection with problems in quantum field theory. Furthermore, the recent work of Kallianpur and Bromley [23] and Kallianpur, Kannan, and Karandikar [24] has brought the Fresnel and Feynman integrals into close contact with the theory of Gaussian stochastic process $[14,22,26]$. We note that $H_{1}$ is the so-called 'reproducing kernel Hilbert space' (or RKHS) $[22,25 ; 26$, pp. 86-91; 28] associated with the Brownian motion stochastic process on $[0, t]$ except that, following $[3]$, the paths vanish at $t$ rather than at 0 . By taking $H$ to be the RKHS for other Gaussian processes, further corollaries similar to those given earlier are easily obtained. The RKHS $\mathrm{H}_{2}=$ $H_{2}[0, t]$ corresponding to the Brownian bridge process $[25$, p. 753$]$ provides a simple example; $H_{2}$ consists of the functions $\gamma$ on $[0, t]$ which are absolutely continuous, 
which vanish at 0 and $t$, and whose derivative $D \gamma$ is in $L^{2}[0, t]$. The inner product on $\mathrm{H}_{2}$ is given by

$$
(\gamma, \beta)_{H_{2}}:=\int_{0}^{t}(D \gamma)(s)(D \beta)(s) d s .
$$

The family of functions $\left\{\gamma_{\tau}: 0 \leq \tau \leq t\right\}$ from $H_{2}$ defined by

$$
\gamma_{\tau}(s):=\min (\tau, s)-s \tau / t
$$

has the reproducing property

$$
\left(\gamma, \gamma_{\tau}\right)_{H_{2}}=\gamma(\tau) \quad \text { for all } \gamma \text { in } H_{2},
$$

and so can play the role played earlier in $H_{1}$ by the functions defined in (3.2).

'Generalized Gaussian processes' provide further settings in which Theorem 1 can be applied. The general framework $[14 ; 22$, pp. 73-74] starts with a Gaussian measure on the dual of a locally convex linear topological space over $\mathbf{R}$. We will just discuss briefly the form taken by Theorem 1 for one particular important example, Gaussian white noise on the space $S^{\prime}$ of tempered distributions or not of $\mathbf{R}$. The RKHS for Gaussian white noise can be identified with $L^{2}(\mathbf{R})[22$, p. 74] and the appropriate parameter space $Y$ is just the Schwartz space $S$ of rapidly decreasing functions on $\mathbf{R}$. The $\phi_{i}$ 's $(i=1, \ldots, d)$ from Theorem 1 become measurable maps from $S$ to $L^{2}(\mathbf{R})$ and the key assumption on $\theta: S \times \mathbf{R}^{d} \rightarrow \mathbf{C}$ is that $\theta(g ; \cdot)=\hat{\nu}_{g}(\cdot)$ where $\nu_{g}$ is in $M\left(\mathbf{R}^{d}\right)$ for every $g$ in $S$. The function $f: L^{2}(\mathbf{R}) \rightarrow \mathbf{C}$ from Theorem 1 then takes the form $f(\psi)=\int_{S} \theta\left(g ;\left\langle\left(\psi, \phi_{1}(g) t\right), \ldots,\left(\psi, \phi_{d}(g)\right)\right\rangle\right) d \eta(g)$ where $\eta$ is a measure on $(S, B(S))$ as in (2) of Theorem 1 .

Another setting of interest is the infinite-dimensional Ornstein-Uhlenbeck process which arises naturally in quantum field theory [4]. Johnson and Kallianpur are hoping to investigate the ramifications of $[\mathbf{2 3}, \mathbf{2 4}]$ and Theorem 1 above for that process.

We will state formally one last corollary. It is the analog of Corollary 5 for the RKHS associated with two-parameter Brownian motion. Let $H_{3}$ denote the set of all functions $\gamma:[0, t]^{2} \rightarrow \mathbf{R}$ for which there exists $v$ in $L^{2}\left([0, t]^{2}\right)$ such that for all $\left(s_{1}, s_{2}\right)$ in $[0, t]^{2}$,

$$
\gamma\left(s_{1}, s_{2}\right)=\int_{0}^{s_{1}} \int_{0}^{s_{2}} v\left(\tau_{1}, \tau_{2}\right) d \tau_{1} d \tau_{2}
$$

It is apparent that $\gamma\left(0, s_{2}\right)=\gamma\left(s_{1}, 0\right)=0$ for all $s_{1}$ and $s_{2}$ and it is not hard to show that $\partial^{2} \gamma / \partial s_{1} \partial s_{2}$ exists and equals $v\left(s_{1}, s_{2}\right)$ for almost every $\left(s_{1}, s_{2}\right)$ in $[0, t]^{2}$. The inner product on $H_{3}$ is defined by

$$
(\gamma, \beta)_{H_{3}}:=\int_{0}^{t} \int_{0}^{t}\left(\frac{\partial 2 s \gamma}{\partial s_{1} \partial s_{2}}\right)\left(\frac{\partial^{2} \beta}{\partial s_{1} \partial s_{2}}\right) d s_{1} d s_{2} .
$$

$H_{3}$, equipped with this inner product, is a separable infinite-dimensional Hilbert space over $\mathbf{R}$ The family of functions $\left\{\gamma_{\tau_{1}, \tau_{2}}:\left(\tau_{1}, \tau_{2}\right)\right.$ is in $\left.[0, t]^{2}\right\}$ from $H_{3}$ defined by

$$
\gamma_{\tau_{1}, \tau_{2}}\left(s_{1}, s_{2}\right):=\left[\min \left(s_{1}, \tau_{1}\right)\right] \cdot\left[\min \left(s_{2}, \tau_{2}\right)\right]
$$

has the reproducing property

$$
\left(\gamma, \gamma_{\tau_{1}, \tau_{2}}\right)_{H_{3}}=\delta\left(\tau_{1}, \tau_{2}\right) \quad \text { for all } \gamma \text { in } H_{3}
$$


COROLlaRY 8. Let $\theta:[0, t]^{2} \times \mathbf{R} \rightarrow \mathbf{C}$ be given by $\theta\left(\tau_{1}, \tau_{2} ; \cdot\right)=\hat{\nu}_{\tau_{1}, \tau_{2}}(\cdot)$ where $\nu_{\tau_{1}, \tau_{2}}$ is in $M(\mathbf{R})$ for every $\left(\tau_{1}, \tau_{2}\right)$ in $[0, t]^{2}$ and where the family $\left\{\nu_{\tau_{1}, \tau_{2}}:\left(\tau_{1}, \tau_{2}\right)\right.$ in $\left.[0, t]^{2}\right\}$ satisfies: (i) $\nu_{\tau_{1}, \tau_{2}}(B)$ is a Borel measurable function of $\left(\tau_{1}, \tau_{2}\right)$ for every $B$ in $B(\mathbf{R})$, and (ii) $\left\|\nu_{\tau_{1}, \tau_{2}}\right\|$ is integrable over $[0, t]^{2}$ with respect to $|\eta|$ where $\eta$ is a Borel measure on $[0, t]^{2}$. Define $f_{8}: H_{3} \rightarrow \mathbf{C}$ by

$$
f_{8}(\gamma):=\int_{0}^{t} \int_{0}^{t} \theta\left(\tau_{1}, \tau_{2} ; \gamma\left(\tau_{1}, \tau_{2}\right)\right) d \eta\left(\tau_{1}, \tau_{2}\right)
$$

Then $f_{8}$ belongs to $\mathcal{F}\left(H_{3}\right)$.

Proof. Apply Theorem 1 with $H=H_{3}, Y=[0, t]^{2}, y=B\left([0, t]^{2}\right), d=1$, and $\phi_{1}\left(\tau_{1}, \tau_{2}\right)=\gamma_{\tau_{1}, \tau_{2}}$ where $\gamma_{\tau_{1}, \tau_{2}}$ is given by (3.15).

We end with a few additional remarks.

1. Many theorems in the monograph [3] and in the more recent literature, for example [11 or 24], involve the assumption that functions belong to $\mathcal{F}(H)$. All such theorems are now seen to apply to the various functions identified in this paper.

2. Theorem 1 is primarily aimed at functions of interest in connection with the Feynman integral and quantum theory. It is of course relevant to, but does not directly address, the larger question of the contents of $\mathcal{F}(H)$. In this connection, we mention that the functions in $\mathcal{F}(H)$ are necessarily bounded and uniformly continuous; in fact, they are even continuous with respect to a topology on $H$ which is coarser than the norm topology [2, p. 10].

3. In what was close to the present version of Theorem 1 , we had assumed that $Y$ was a topological space and that $Y$ was the Borel class of $Y$. In that case, we had the added assumption that $B\left(Y \times \mathbf{R}^{d}\right)=B(Y) \times B\left(\mathbf{R}^{d}\right)$. Kallianpur kindly pointed out to one of us that we could just as well work with an abstract measurable space $(Y, y)$ and the product $\sigma$-algebra $y \times B\left(\mathbf{R}^{d}\right)$ on $Y \times \mathbf{R}^{d}$.

4. Because of the close relationship between $\mathcal{F}\left(H_{1}\right)$ and an analogous Banach algebra of functions on Wiener space [15] and, more generally, between $\mathcal{f}(H)$ and the 'Fresnel class' $F(B)$ of functions on the 'abstract Wiener space' $B$ associated with $H[23,24,26]$, one would guess that results analogous to Theorem 1 ought to be true in these settings. We have substantial progress in this direction and conjecture that the full results hold.

\section{REFERENCES}

1. S. Albeverio, Ph. Blanchard, and R. Høegh-Krohn, Feymman path integrals and the trace formula for the Schrödinger operators, Comm. Math. Phys. 83 (1982), 49-76.

2. S. Albeverio and R. Høegh-Krohn, Feymman path integrals and the corresponding method of stationary phase, Feynman Path Integrals, Marseille, 1978, Lecture Notes in Physics, vol. 106, Springer, Berlin, 1978, pp. 3-57.

3. __ Mathematical theory of Feynman path integrals, Lecture Notes in Math., vol. 523, Springer, Berlin, 1976.

4. J. Bertrand and B. Gaveau, Transformation canonique et renormalization pour certaines équations d'évolution, J. Funct. Anal. 50 (1983), 81-99.

5. R. H. Cameron and D. A. Storvick, Some Banach algebras of analytic Feymman integrable functions, Analytic Functions, Kozubnik, 1979, Lecture Notes in Math., vol. 798, Springer, Berlin, 1980, pp. 18-67. 
6. $\ldots$, A simple definition of the Feymman intergral, with applications, Mem. Amer. Math. Soc. No. 288, vol. 46, 1983, pp. 1-46.

7. K. S. Chang, G. W. Johnson, and D. L. Skoug, Necessary and sufficient conditions for the Fresnel integrability of certain classes of functions, J. Korean Math. Soc. 21 (1984), 21-29.

8. 122 (1986), 11-33.

9. A. M. Chebotarev, $\tau$-mappings and functional integrals, Soviet Math. Dokl. 16 (1975), 15361540 .

10. Ph. Combe, R. Høegh-Krohn, R. Rodriguez, M. Siruque, and M. Siruque-Collin, Poisson processes on groups and Feymman path integrals, Comm. Math. Phys. 77 (1980), 269-288.

11. D. Elworthy and A. Truman, Feymman maps, Cameron-Martin formulae and anharmonic oscillators, Ann. Inst. H. Poincaré 41 (1984), 115-142.

12. P. Exner, Open quantum systems and Feymman integrals, Reidel, Dordrecht, 1985.

13. R. P. Feynman, Space-time approach to non-relativistic quantum mechanics, Rev. Mod. Phys. 20 (1948), 367-387.

14. T. Hida, Brownian motion, Springer, New York, 1980.

15. G. W. Johnson, The equivalence of two approaches to the Feymman integral, J. Math. Phys. 23 (1982), 2090-2096.

16. __ An unsymmetric Fubini theorem, Amer. Math. Monthly 91 (1984), 131-133.

17. G. W. Johnson and M. L. Lapidus, Generalized Dyson series, generalized Feymman diagrams, the Feymman integral, and Feynman's operational calculus, Mem. Amer. Math. Soc. No. 351, vol. 62, 1986, pp. 1-78.

18. G. W. Johnson and D. L. Skoug, Notes on the Feymman integral. I, Pacific J. Math. 93 (1981), 313-324.

19. __ Notes on the Feynman integral. II, J. Funct. Anal. 41 (1981), 277-289.

20. __ Notes on the Feymman integral. III: The Schroedinger equation, Pacific J. Math. 105 (1983), 321-358.

21. __ Stability theorems for the Feymman integral, Suppl. Rendi. Circ. Mat. Palermo (Proc. Conf., Palermo, October, 1984) (to appear).

22. G. Kallianpur, The role of reproducing kernel Hilbert spaces in the study of Gaussian processes, Advances in Probability, Vol. 2 (Peter Ney, editor), Dekker, New York, 1970, pp. 49-83.

23. G. Kallianpur and C. Bromley, Generalized Feynman integrals using analytic continuation in several complex variables, Stochastic Analysis and Applications (M.A. Pinsky, editor), Dekker, New York, 1984.

24. G. Kallianpur, D. Kannan, and R. L. Karandikar, Analytic and sequential Feymman integrals on abstract Wiener and Hilbert spaces and a Camenon-Martin formula, Ann. Inst. H. Poincaré 21 (1985), 323-361.

25. J. Kuelbs, $A$ strong convergence theorem for Banach space valued random variables, Ann. Probab. 4 (1976), 744-771.

26. H.-H. Kuo, Gaussian measures in Banach spaces, Lecture Notes in Math., vol. 463, Springer, New York, 1975.

27. M. L. Lapidus, The differential equation for the Feymman-Kac formula with a Lebesgue-Stieltjes measure, Lett. Math. Phys. 11 (1986), 1-13.

28. J. Neveu, Mathematical foundations of the calculus of probability, Holden-Day, San Francisco, Calif., 1965.

29. C. Park and D. L. Skoug, The Feymman integral of quadratic potentials depending on $n$ time variables (submitted for publication).

30. W. Rudin, Real and complex analysis, McGraw-Hill, New York, 1966.

31. L. Streit and T. Hida, Generalized Brownian functionals and the Feymman integral, Stochastic Process. Appl. 16 (1984), 1-15.

\section{Department of MAthematics, Yonsei University, SeOUl, Korea}

Department of MAThematics and Statistics, UNiversity of Nebraska, LiNCOLN, NEBRASKA 68588 